ENCYCLOPÉDIE Encyclopédie berbère

BERBERE

$31 \mid 2010$

31 | Matmora - Meẓrag

\title{
Maurétanie (Royaumes)
}

M. Coltelloni-Trannoy

\section{OpenEdition}

Journals

Édition électronique

URL : http://journals.openedition.org/encyclopedieberbere/521

DOI : 10.4000/encyclopedieberbere.521

ISSN : 2262-7197

\section{Éditeur}

Peeters Publishers

\section{Édition imprimée}

Date de publication : 30 décembre 2010

Pagination : 4717-4737

ISBN : 978-90-429-2368-3

ISSN : 1015-7344

\section{Référence électronique}

M. Coltelloni-Trannoy, « Maurétanie (Royaumes) », Encyclopédie berbère [En ligne], 31 | 2010, document M69a, mis en ligne le 08 octobre 2020, consulté le 12 octobre 2020. URL : http://

journals.openedition.org/encyclopedieberbere/521 ; DOI : https://doi.org/10.4000/ encyclopedieberbere.521

Ce document a été généré automatiquement le 12 octobre 2020

(c) Tous droits réservés 


\section{Maurétanie (Royaumes)}

\section{Coltelloni-Trannoy}

1 L'hésitation même qui porte à user du singulier, «la Maurétanie ", comme du pluriel, «les royaumes", met en évidence les difficultés de la question: d'une part, «la Maurétanie » a bel et bien existé, en tant que réalité opposée à divers titres (culturel, géographique, politique) à la Numidie; d'autre part, le singulier sous entend une unité et une stabilité que les sources n'incitent guère à observer, quelle que soit l'approche choisie, ethnique, politique ou territoriale. Deux problèmes se posent, qui sont en étroite connexion. Le premier concerne la transformation de la gens Maurorum en un regnum Maurorum ou Mauretaniae: il s'agit d'un processus qui ne peut se comprendre qu'en relation avec les puissances du temps, Carthage, les royaumes hellénistiques et l'Empire romain. Mais par ailleurs, cette transformation n'offre pas un visage linéaire ni simple : il exista plusieurs royaumes de Maurétanie ou « des Maures», successifs ou simultanés, distincts des royaumes numides, ou bien au contraire les englobant. En sa dernière phase, le royaume de Maurétanie, qui est une création de l'empereur Auguste, s'étend sur un immense territoire avant que Caligula n'en décide l'annexion et que Claude ne le répartisse en deux provinces (en 42), la Maurétanie Césarienne et la Maurétanie Tingitane. Une réalité territoriale mouvante et qui ne fut jamais clairement définie, un pouvoir en mutation sur deux siècles : telles sont les difficultés auxquelles se heurte qui tente de comprendre, de décrire et d'expliquer la genèse et l'évolution de ces royaumes à l'époque préromaine.

2 Les efforts des historiens depuis S. Gsell ont permis de reconstituer dans leurs grands traits l'histoire et la géographie des royaumes maures, jusqu'à la Mauretania de Juba II et de Ptolémée. Bien des lacunes subsistent en raison du caractère approximatif et irrégulier de notre documentation: les doutes portent sur la filiation exacte des dynasties, sur l'évolution territoriale des royaumes (y compris dans leur partie méditerranéenne, la mieux connue), sur la succession des événements, notamment entre l'achèvement de la guerre de Jugurtha (105) et la guerre qui opposa en Afrique César aux pompéiens (46). Néanmoins, on observe bien que cette histoire eut pour caractéristique le glissement vers l'est de la domination maure, à partir du II ${ }^{\mathrm{e}}$ siècle, qui alla jusqu'à englober la majeure partie des anciens royaumes numides. Cette histoire est aussi celle du pouvoir royal et de l'enracinement territorial des royaumes qui 
échappèrent progressivement (mais jamais totalement) à la logique tribale sous l'influence du modèle hellénistique et de la présence romaine en Afrique ; c'est enfin l'histoire de leur ouverture au monde méditerranéen.

\section{Une histoire mal connue des historiens anciens}

3 Nombreuses sont les erreurs ou les approximations que les auteurs anciens ont commises à propos des différentes phases de la Maurétanie, y compris la dernière, la mieux connue d'eux pourtant, le royaume de Juba II et de Ptolémée. Elles s'expliquent aisément par l'histoire complexe de cette région qui entraîna remaniements territoriaux et changements dynastiques sur environ deux siècles. Les sources impériales ont gardé la trace de ces mutations, se référant souvent de manière indistincte à un état territorial ou à une ethnie dominante sans grand respect pour la chronologie des faits et sans parvenir à donner sa cohérence à un savoir éclaté. Mais au-delà de ces erreurs historiques qu'il faut imputer à des informations partielles et mal maitrisées, on observe que ces contrées sont longtemps demeurées - et ce, en pleine époque romaine - empreintes de mystère et de merveilleux. On les intégrait plus volontiers dans les mythes que dans un savoir scientifique, et le fantastique que l'on aimait rencontrer dans les récits de confins consacrés à l'Afrique (la Libya des Grecs), tels ceux d'Homère, d'Hérodote et des périples grecs, trouve encore un écho dans certaines évocations de Salluste ou de Lucain, mais aussi dans les fragments des œuvres du roi Juba relatifs à l'Afrique. L'éloignement de ces zones a même suscité la conception de deux Tingitane, une qualifiée de Mauretania extuma ou ulterior (une Maurétanie de "l'au-delà »), l'autre de Mauretania citerior, celle du littoral méditerranéen, sans doute réputée être mieux connue et plus proche des normes de vie romaines (Pline, Histoire naturelle, $\mathrm{V}: 19$; Desanges 1960).

Une seconde strate mythique apparut à une date plus récente, lorsque les Romains, les Grecs, mais aussi les Africains eux-mêmes (en témoignent les écrits de Hiempsal II et de Juba II : cf infra) s'attachèrent à insérer les Maures et les Numides dans l'histoire des peuples méditerranéens et surtout dans leurs mythes. On en a un aperçu dans la première relation détaillée de l'histoire africaine dont nous disposions, transmise par Salluste (Guerre de Jugurtha, XVIII) qui tire ses informations des livres du roi numide Hiempsal, son contemporain : l'origine biologique des peuples maures et numides est mise en relation avec les compagnons d'Hercule, dont on sait qu'il combinait des traits grecs, latins et phénico-puniques (Melqart). C'est ainsi que les Mèdes et les Perses, qui constituaient l'armée d'Hercule, mort en Espagne, se mêlèrent, les premiers avec les Libyens pour constituer le peuple des Maures, et les seconds avec les Gétules, devenant alors les Nomades, puis les Numides. L'élaboration fabuleuse de ces généalogies mélange à la fois une constante de l'imaginaire antique (l'origine orientale des peuples ou des cultures) et un goût marqué pour les calembours étymologiques. Par la suite, plusieurs autres légendes ont toutes assigné une origine orientale aux Africains, en l'intégrant souvent au mythe d'Hercule. Deux d'entre elles, dues à Plutarque (Sertorius, $9,4,5)$ et à Flavius Josephe (Antiquités juives, I : 15), ont pour point commun de s'intéresser de près aux généalogies mythiques des dynasties africaines et de donner une importance première au rameau occidental, celui des Maures: la veuve du géant Antée, Tingè (éponyme de Tingi), aurait eu un fils avec Hercule, nommé Sophax, luimême père de Diodoros, l'ancêtre des dynasties massyle et masaesyle (Josèphe inverse 
la place de Deodoros et de Sophax). Il est vraisemblable que ces spéculations mythologiques ont été imaginées par le roi Juba II et qu'il poursuivait ainsi un double objectif : conférer une origine prestigieuse (herculéenne) à sa lignée, sur le modèle de la généalogie vénusienne élaborée par César et par Auguste; légitimer sa domination sur la partie occidentale et la partie orientale de son royaume, en introduisant un lien naturel et historique entre les deux territoires, leurs peuples et leurs dynasties.

C'est que ce lien n'allait effectivement pas de soi : le dernier royaume de Maurétanie était le résultat de la volonté d'Auguste, qui réorganisa l'ensemble de l'Afrique mineure en deux grandes entités territoriales et politiques (province d'Afrique proconsulaire et royaume de Juba II).

6 Strabon, qui fut un contemporain de Juba II, est sensible au caractère unitaire du vaste royaume qu'Auguste avait confié au roi (XVII, 3, 12 et 3,24), mais certains descriptifs n'en sont pas moins problématiques: un premier passage de sa Géographie (VI, 4, 2) évoque, en effet, Juba comme étant le roi de la Maurousie (la forme grecque de Mauretania) et d'une partie de la «Libye » tandis qu'un autre passage (XVII, 3, 7) fait état d'un ancien royaume maure, unique, celui de Bogud et de Bocchus, auquel Auguste adjoignit le royaume numide de Juba I, le père de Juba II. Par conséquent, le dernier royaume de Maurétanie, est, aux yeux de Strabon, le conglomérat tardif d'un royaume maure précédemment unifié (mais sous l'autorité de deux rois) et d'un territoire numide (en réalité une fraction du royaume de Juba I, la partie orientale ayant été réunie à la Proconsulaire). Il y a mieux: oubliant totalement ces assertions, le géographe base peu après un panorama global de la région sur des informations datant de deux siècles, en évoquant le territoire des Maures et celui des Masaesyles, qu'il situe entre la Moulouya et le Cap Bougaroun, c'est-à-dire les Numides occidentaux à l'époque de Syphax! (XVII, 3, 9). Ailleurs encore (VII, 3, 12), il indique que la colonie romaine de Saldae, située sur la Saua (Soummam), est à la limite qui sépare le royaume de Juba de la province : or, cette limite est obsolète, puisque c'était celle d'un état un plus ancien de la Maurétanie, celui du royaume de Bocchus II (contigu à celui de Juba I).

7 Si l'on se tourne à présent vers un autre témoin, Pline l'Ancien, qui eut à sa disposition des informations solides sur la Maurétanie, du fait même qu'il fut contemporain de la réorganisation des deux provinces maurétaniennes, sous Vespasien, et qu'il exerça des fonctions procuratoriennes dans la province voisine, la Bétique, on s'aperçoit que sa relation est également embarrassée : s'il parle avec exactitude de la Mauretania du roi Ptolémée $(\mathrm{HN}, \mathrm{V}, 11)$, il se réfère aussi aux deux Maurétanies (utrique Mauretaniae, $\mathrm{V}, 16)$ sur lesquelles auraient régné Juba et son fils Ptolémée : « Les premières terres que l'on rencontre portent le nom de Maurétanies, royaumes jusqu'à $C$. César, fils de Germanicus, puis divisées par l'effet de sa cruauté en deux provinces » $(\mathrm{V}, 2,1)$. Ce faisant, il sous-entend que la création des deux provinces se justifiait par la composition même du royaume, qu'il considère comme ayant été bifide, regroupant en quelque sorte deux royaumes de Maurétanie! Sa position est erronée, tout comme l'était celle de Strabon qui, lui, établissait une continuité entre la création augustéenne et la Maurétanie antérieure. L'un et l'autre essayaient d'expliquer les décisions du moment (celle d'Auguste puis celle de Claude) par le maintien d'une situation antérieure; l'un et l'autre se trompaient. 


\section{De la gens aux regna : l'évolution territoriale et dynastique}

8 Les dénominations de Maures, Numides, Massyles, Masaesyles désignaient à l'origine non pas des territoires, mais des tribus ou des confédérations de tribus. Loin d'être des entités ethniques stables, elles formaient des groupements de peuples qui étaient constamment ouverts à de nouvelles ethnies et qui s'adaptaient aux réalités historiques. Les dénominations Maurousia ou Mauretania qui indiquent l'existence d'une entité territoriale et dynastique stable apparaissent tardivement, au I ${ }^{\mathrm{er}}$ siècle av. J.-C. ; elles sont toujours restées concurrencées par des formules plus anciennes qui mettaient l'accent sur les Maures ou le roi des Maures, c'est-à-dire sur le peuple et non sur un espace (plus ou moins) défini. La même observation pourrait être faite à propos de la Numidia et des rois numides. D'après les sources littéraires, l'existence de royaumes maures et numides est un fait avéré au III ${ }^{e}$ siècle, ce qui signifie que ces royaumes, ou du moins de vastes confédérations de tribus, se sont constitués à une époque antérieure qu'il est malaisé de préciser, sans doute au cours du IV ${ }^{\mathrm{e}} \mathrm{s}$. (Camps 1960). Les Maures, dont le nom (Mauri) est sans doute d'origine locale (Strabon, XVII, 3 , 2), occupaient à l'origine le nord du Maroc, aux alentours du détroit de Gibraltar, et en formaient la principale tribu (Pline, $H N, \mathrm{~V}, 17,2$ ). Cependant, le roi Baga, qui est le premier à nous être connu (III ${ }^{\mathrm{e}} \mathrm{s}$.), étendait son autorité aux régions voisines de la Masaesylie puisqu'il soutint Massinissa contre Syphax. La mention d'un ou de deux royaumes maures situés dans la région et la ressemblance des noms royaux (Baga, Bocchus, Bogud) suggèrent que la même dynastie régna jusqu'au Ier siècle av. J.-C. Leur assise territoriale demeurait toutefois fragile et la puissance des rois surtout fondée sur leur capacité à mobiliser le soutien des chefs de tribus et de cités: Bocchus l'Ancien prétendait être « le plus grand roi de cette terre et de tous les rois [que je connaisse] » (Sall., Jug., CX, 1), une affirmation qui le plaçait, au Maroc, en position dominante par rapport à d'autres sources d'autorité locales (cités et tribus); la présence de plusieurs grands tumuli, datés des $\mathrm{III}^{\mathrm{e}} / \mathrm{I}^{\mathrm{er}}$ siècle av. J.-C., témoigne que de puissants personnages étendaient leur pouvoir autour de Ksar-el-Kebir, Gilda et Volubilis; la partition du royaume entre Bogud et Bocchus (II) et la présence d'une principauté vassale à Tingi (Plut., Sert., 9) sont aussi les indices d'une unité encore en devenir au $\mathrm{I}^{\mathrm{er}}$ siècle.

Ceci met en lumière une autre caractéristique de ces royaumes, où le pouvoir demeura longtemps tributaire des structures tribales. La définition du pouvoir tribal et de sa logique de transmission dans les sociétés proto-berbères s'appuie sur les rares indices laissés par les sources anciennes, ainsi que sur les résultats de l'ethnologie. S. Gsell (HAAN, V : 121-122) en a très clairement énoncé les principes : "la royauté était la propriété d'une famille, dans le sens large du terme, c'est-à-dire d'un ensemble d'agnats remontant par les mâles à un ancêtre commun [...]. Dans cette famille, le chef était le plus âgé des mâles vivants issus de mariages légitimes. A lui appartenait la royauté. A sa mort, elle passait à celui qui devenait l'aîné du groupe d'agnats ». Cette logique semble vérifiée pour la lignée de Massinissa, jusqu'à ce roi (Camps 2007 : 103-104), mais, à la période ultérieure, elle entre en concurrence avec une autre règle que Scipion imposa aux fils de Massinissa: elle semble combiner le principe de la filiation et une règle, peut-être d'origine libyque, voulant que l'autorité royale ou le territoire soient répartis entre deux ou trois souverains. Ainsi se réglèrent à la suite de 
la succession de Massinissa, celle de Micipsa chez les Numides et celle de Sosus ( ?) chez les Maures.

10 Le principe de filiation finit par s'imposer au début du $\mathrm{I}^{\mathrm{er}}$ siècle av. J.-C. dans les royaumes numides, sur le modèle des royautés hellénistiques. Côté maure, il n'est pas attesté en toute certitude avant Juba II bien que l'existence d'un roi Sosus, père ( ?) de Bocchus II et de Bogud soit vraisemblable; quant au roi Bocchus II, il éprouva la nécessité de mentionner son père (qu'il fût roi ou pas) sur ses monnaies (Sosi filius), imitant ainsi les monnaies grecques et numides, et revendiquant une légitimité fondée sur le modèle de succession par la filiation. Ce principe est naturellement adopté par Juba II qu'une inscription de Carthagène inscrit dans une longue généalogie royale, jusqu'à Massinissa, considéré à tort comme le père de Gauda (CIL, II, $3417=$ ILS, 840). Cette généalogie occulte complètement la distorsion qui existait entre la réalité dynastique et la réalité territoriale ou politique : prince de sang numide, Juba II régnait sur un royaume qui s'était constitué grâce aux efforts des derniers rois maures et que ni son père ni ses aïeux (sauf Massinissa) n'avaient dominé. Il associa son fils Ptolémée à son pouvoir, en 19, selon une pratique usuelle aux rois hellénistiques ; peut-être fautil y voir aussi une touche romaine, sachant que les empereurs Auguste et Tibère, tous deux soucieux de renforcer leur légitimité, avaient choisi pour collègues des hommes de leur famille après les avoir adoptés.

11 Le premier roi maure connu, Baga, est cité par Tite-Live comme ayant soutenu Massinissa, une première fois en lui donnant une escorte de 4000 guerriers contre Syphax (XXIX, 29, 7), et une seconde en envoyant des contingents combattre Carthage (XXIX, 30, 3). Mais les Romains n'entrent en relation directe avec les Maures qu'à l'occasion de la guerre de Jugurtha, quand Sylla entreprend de rompre l'alliance qui unissait le roi numide à son beau-père, le roi maure Bocchus (I). Ce dernier finit par accepter de trahir Jugurtha, contre la promesse de récupérer une partie de son royaume, une promesse que les Romains respectèrent effectivement après la capture de Jugurtha, en 105 (Sall., Jug., CXI, 2). On ignore l'ampleur exacte de cette première extension, peut-être le tiers que lui avait promis Jugurtha (id., XCXVII, 2), ce qui aurait amené la limite du royaume au Cheliff.

12 A partir de là s'amorça la série des empiètements qui en moins d'un siècle fit des Maures la puissance dominante en Afrique. Toutefois, la succession dynastique fait toujours débat. Pour G. Camps (EB, B84 : 1544 sq), Bocchus a transmis son royaume à un Sosus (son fils?), dont l'existence (80 ?-49 ?) serait attestée par plusieurs indices : des monnaies dont la légende en néopunique donnerait son titre, MSTNS (contra: Alexandropoulos, $\mathrm{n}^{\circ} 41$ : 189-190); la mention de son nom sur les monnaies d'un autre Bocchus (II), donnant en latin la filiation paternelle, Sosi f(ilius) (Alexandropoulos, $\mathrm{n}^{\circ}$ 60-63) ; l'allusion de Cicéron (In Vatinium, 5, 12) à un Mastanesosus pourrait recouvrir le nom du roi, associé à son titre en libyque, Mastan; enfin la découverte de balles de fronde recueillies à Volubilis, portant la marque REX SOS.

Deux rois maures, frères ou cousins, sont ensuite mieux connus; ils opèrent une partition du royaume maure, de part et d'autre de la Moulouya : Bocchus (II) roi de Maurétanie orientale (49-33) et Bogud (49-38), roi de Maurétanie occidentale, tous deux en place au moins en 49. Contraints sans doute de choisir leur camp au moment des guerres civiles, en raison des liens de clientèle qui les liaient aux puissantes familles romaines, l'un et l'autre font d'abord en 49 le même choix, celui du parti césarien. Si Bogud resta prudemment extérieur aux opérations militaires du moment, Bocchus, 
dont le royaume était voisin de ceux du roi numide Juba I et de son vassal Masinissa, alliés aux Pompéiens, joua un rôle déterminant. Il s'était, en effet, adjoint les services d'un chef militaire italien, Sittius, chargé d'organiser et d'entraîner son armée «à la romaine » : tous deux apportèrent une aide précieuse à César en attaquant le territoire de Juba I, ce dont ils furent récompensés en 46 en récupérant chacun une partie du royaume numide démembré : Sittius eut la région de Cirta (annexée à l'Africa à la mort de Sittius, en 44) et Bocchus la partie occidentale de la Numidie, jusqu'à l'Ampsaga (oued el-Kebir), tandis que la partie orientale était annexée à l'Empire (Africa noua). Les choses n'en restèrent pas là. La guerre entre Antoine et Octave eut à son tour des répercussions sensibles en Afrique, d'ordre politique et territorial : dès 38 , les deux rois entrèrent dans les camps opposés (Bogud pour Antoine et Bocchus pour Octave); peu après, Bocchus et les césariens soutinrent une révolte de Tingi, privant ainsi Bogud de son royaume dont Bocchus s'empara (Dion Cassius, 45, 1-3). Tingi fut récompensée de son soutien actif par l'octroi du statut municipal qui annonçait la série des fondations coloniales d'Auguste dans le royaume.

La mort de Bocchus, en 33, suivie de près par celle de Bogud à la bataille de Méthone, en 31, laissait vacant un immense territoire, aux portes de la province d'Afrique: ce royaume sans rois portait le nom de Maurétanie en raison de l'ethnie à laquelle avaient appartenu ses derniers souverains, mais correspondait, sur ses deux tiers, à des régions de peuplement numide; ses limites le portaient désormais jusqu'à l'Ampsaga (oued elKebir), qui devait rester, jusqu'à la fin de l'Empire, la limite septentrionale séparant la Maurétanie de la province d'Afrique. De 33 à 25, le territoire connut une situation d'attente car, s'il fut bien compté « au nombre des provinces romaines » (DC, 49, 43, 7), si l'atelier de Iol (Cherchel) émit des monnaies au nom d'Octave, si ce dernier y fonda pas moins de douze colonies (outre Tingi qui était municipe depuis 38), si enfin la population de Zilil subit un transfert autoritaire en Espagne (Strabon, III, 2, 8), aucune administration provinciale complète n'y fut installée: ni gouverneur, ni armée, ni services fiscaux. C'est une période que les historiens ont coutume d'appeler «l'interrègne » puisque Auguste y mit fin en 25, installant dans la capitale, Iol, bientôt rebaptisée Caesarea, une nouvelle dynastie qui ne devait rien aux rois maures antérieurs. Non seulement son roi était le fils du roi numide Juba $\mathrm{I}^{\mathrm{er}}$, mais son épouse, Cléopâtre Séléné, fille de Cléopâtre VII d'Egypte, était apparentée à la maison impériale par son père, Marc Antoine. La réalité territoriale elle-même avait connu un certain remaniement : au noyau maurétanien de Bocchus, l'empereur avait adjoint une fraction du royaume de Juba $\mathrm{I}^{\text {er }}$.

Le dernier royaume de Maurétanie s'étendait sur un territoire bien délimité au nord par des accidents naturels (l'Atlantique, la Méditerranée, le fleuve de l'Ampsaga), mais qui prenait un caractère indéfini dès que l'on considère ses limites méridionales. Même la délimitation avec la province d'Afrique est douteuse : les territoires gétules soumis au roi, au Sud-Est de l'Ampsaga, s'étendaient-ils bien jusqu'au chott el-Hodna et au fleuve Nigris/ oued Djedi (Desanges 1957 et 1964) ? Et quelle était, au nord, l'extension de cette région gétulique qui se souleva contre Juba et les Romains en 6 ap. J.-C. avant de rejoindre les rangs de l'armée de Tacfarinas? Faut-il penser qu'elle remontait jusqu'à l'actuelle Aumale, souvent identifiée à Auzea que Tacfarinas assiège en 24 (Tac., Annales, IV , 25) ? Quel fut le statut du cantonnement établi par Lentulus pour surveiller les Gétules voisins des Musulames*, suite à la guerre gétulique (Orose, VI, 21, 18)? Etait-il soumis à l'autorité royale ou bien constituait-il un espace intermédiaire, surveillé conjointement par le roi et par les Romains? Ces incertitudes auxquelles il 
convient d'ajouter celles qui touchent à tous les autres confins du royaume ne doivent pas suggérer une différence irréductible avec l'Empire. Le propre du "limes» romain tenait à sa nature mouvante, imprécise, constamment redéfinie par les gains de terre et par des alliances avec les peuples extérieurs et intérieurs : le royaume de Maurétanie offrait précisément les caractères d'une zone romaine de confins, d'un limes de la province d'Afrique. A ce titre, il s'intégrait bien à l'économie générale de l'Empire.

Lorsque le fils de Juba, Ptolémée, fut exécuté sur ordre de Caligula (fin 39), le royaume fut annexé (41) avant d'être réparti entre deux provinces de Maurétanie, la Césarienne et la Tingitane (42). Leur territoire respectif reproduisait, cette fois, la configuration qui jadis avait réparti, autour de la Moulouya, les Numides et les Maures: le choix de Claude s'appuyait sur des considérations géographiques et ethniques qui avaient paru secondaires au temps d'Auguste. Les oscillations que l'on observe dans la représentation que les Romains se faisaient de la Maurétanie (unifiée ou bipartite) ne doivent pas occulter un fait d'importance: l'enracinement territorial des royaumes africains, notamment la Maurétanie, n'a cessé de se confirmer du fait de l'évolution même du pouvoir royal et des interventions des Romains dans l'histoire africaine.

\section{Les mutations du pouvoir royal}

Nous disposons de deux groupes de sources pour évaluer la transformation du pouvoir royal vers un pouvoir de type hellénistique: les sources externes, c'est-à-dire les relations que les Grecs et les Romains ont laissées à ce propos; les sources internes, produites par les rois eux-mêmes (monnayages, écrits) ou bien issues de leurs royaumes (céramique, épigraphie). Un simple panorama de ces sources laisse apparaître des décalages significatifs. Un décalage concerne la chronologie selon laquelle se met en place le processus de monétarisation des pouvoirs africains, bien étudié par J. Alexandropoulos : ce processus, qui permet aux rois de se représenter en s'inspirant des normes hellénistiques puis romaines, est plus précoce en Numidie (sous Syphax puis Massinissa) qu'en Maurétanie (sous Bocchus I, Alexandropolos : 193-196), l'écart jouant sur plus d'un siècle. Un autre décalage porte sur les époques qui voient émerger les peuples ou les rois africains dans les sources externes: ils restent cantonnés, nous l'avons déjà vu, dans les récits mythiques pendant une longue période et n'attirent pas l'attention des historiens avant les guerres puniques, au moment où les Romains ont connaissance de leurs liens étroits avec Carthage (la guerre des Mercenaires) ou bien prennent contact directement avec eux (à partir de la deuxième guerre punique). Mais on observe aussi des convergences: convergence entre le moment même où Rome prend pied en Afrique et celui où l'on note les premiers monnayages numides (fin $\mathrm{III}^{\mathrm{e}} \mathrm{s}$ ) ; autre convergence entre la date des premiers monnayages maures et celle des premières relations diplomatiques entre les Romains et Bocchus I. Il est frappant de voir à quel point les nouvelles logiques politiques territoriales se mettent en place en Afrique sous deux impulsions simultanées : à la faveur de l'hellénisation du pouvoir africain, dans laquelle le modèle punique a joué un rôle central, et à la faveur de l'influence romaine.

On distingue à l'origine deux traditions monétaires, tournées chacune vers le monde punique: l'une, maurétanienne, est déterminée par l'Espagne barcide, et surtout Gadès ; l'autre, numide, par le système carthaginois. Puis la réforme du roi Juba I, celle de Bogud ou de Bocchus II introduisent plusieurs éléments d'inspiration romaine: 
l'usage du latin à côté du néopunique, les émissions d'argent, la métrologie ou l'iconographie intègrent ces systèmes monétaires à l'économie romaine, dans le même temps que les deniers romains circulaient en grand nombre en Afrique; ces choix traduisent aussi les options politiques des rois, tous engagés dans les guerres civiles romaines. Les émissions de l'interrègne tentent la première fusion de ces traditions diverses avec l'objectif évident de présenter Rome en héritière des rois africains. Mais c'est aux deux derniers rois de Maurétanie qu'est échue la charge de fondre en un monnayage cohérent cette masse monétaire antérieure, toujours en circulation. Le recours aux trois métaux, la domination du latin qui laisse place à quelques légendes en grec, la grande variété des types de facture romaine, lagide et africaine, la métrologie essentiellement romaine (les bronzes de Juba II sont encore proches du système numide) : tout porte à faire des deux derniers monnayages maurétaniens les blasons d'un pouvoir hellénistique parfaitement intégré au système politique et monétaire romain; en témoigne la longue survivance des monnaies royales dans les provinces romaines.

19 Il est un autre "produit ", plus rare, mais tout aussi significatif de cet affichage du pouvoir royal : les écrits des rois Hiempsal II et Juba II, qui témoignent de l'utilisation de l'écrit comme outil de pouvoir. Bien que le premier ait régné sur une Numidie orientale (88-50 ?) et non sur un royaume de Maurétanie, il convient d'évoquer son action en la matière parce qu'elle atteste une tradition d'érudition et d'écriture en grec parmi les rois africains, aux côtés de son aïeul Mastanabal et de son petit-fils Juba II : on devait à Hiempsal la traduction, voire la réécriture, de certains des livres puniques confiés par Scipion à sa famille après l'incendie de Carthage ; quant à Juba II, son œuvre immense et variée (mais conservée sous forme fragmentaire) a fait de lui l'une des références premières de la science d'époque impériale. L'activité littéraire de ces deux rois n'est pas uniquement la manifestation d'un don personnel, mais aussi celle d'une politique consciemment suivie: les deux rois s'inscrivaient ainsi dans le cercle des auteurs romains, tous issus de grandes familles dont la puissance se mesurait aussi au monopole qu'elles exerçaient sur la culture; d'autre part, leur choix de rédiger leurs œuvres en grec conférait à celles-ci un bon support de communication, apte à toucher toutes les élites du monde méditerranéen, grecques et romaines. En somme, L'un et l'autre se conformaient aux normes du pouvoir attendu de souverains "éclairés », en prétendant donner à leur souveraineté la visibilité la plus large qui fût. En se faisant les promoteurs de la paideia grecque en milieu africain, ils ambitionnaient d'occuper une place éminente parmi les souverains de leur époque, non sans reconnaître la suprématie de Rome dans l'organisation de l'oikoumène.

20 Le renforcement du pouvoir royal se mesure aussi à l'aune de deux autres réalités (outre le processus dynastique vu plus haut): l'émergence de villes regiae et la formation d'armées " modernes ", inspirées des modèles hellénistique et romain.

21 Les villes sont très anciennes en Afrique, du moins dans la zone littorale ou proche $d u$ littoral méditerranéen ou atlantique : si beaucoup sont des créations puniques ou ibéropuniques, on sait que des villes libyennes apparaissent dès avant l'arrivée des Phéniciens, surtout des établissements portuaires (en Maurétanie: Tingi, Lixus, Sala), tandis que les habitats situés dans l'arrière-pays et résultant de la sédentarisation de tribus berbères sont sans doute plus tardifs (Banasa, Volubilis). Les royaumes africains ont donc fédéré, dès leur émergence, sous une même autorité personnelle puis dynastique, un tissu social hétérogène, fait de tribus et de cités préexistantes. La 
prospérité agricole de la Numidie a naturellement facilité le processus d'urbanisation, en particulier dans sa partie orientale qui bénéficiait aussi de la proximité avec Carthage. Dès la fin du III ${ }^{\mathrm{e}}$ siècle, certaines de ces villes se distinguent des autres, soit par leur titre de regia qui indique un lien privilégié avec le pouvoir royal, soit par leur rôle qui présente certaines analogies avec les capitales du monde hellénistique: Siga pour le royaume de Syphax ; Cirta pour celui de Massinissa qui lui adjoint Siga avant que son fils Micipsa ne se recentre à Cirta ; Zama Regia dans la Numidie de Juba I.

Parmi les villes maures, il en est trois qui se détachent tout particulièrement, Volubilis, Lixus et Tingi. Aux deux dernières sont attachés des épisodes de la légende herculéenne et l'on sait que, dès l'époque phénicienne, elles rivalisaient avec leurs consœurs ibériques, notamment Gadès, ayant en commun avec elles une même ouverture sur tout le monde méditerranéen. Quant à Volubilis, elle resta en retrait de ces échanges jusqu'au $\mathrm{III}^{\mathrm{e}} \mathrm{s}$ : à cette époque, non seulement l'urbanisme et les institutions puniques y furent adoptés, mais il est possible qu'à partir du roi Bocchus I, la cité ait abrité un atelier royal (Alexandropoulos 2000 : 197-198), contre l'idée traditionnelle attribuant à Lixus cet atelier : en témoignerait la diffusion très forte de bronzes à la légende $\breve{M}$ Š et portant le nom et l'effigie du roi, dans toute la région, alors qu'ils sont en diminution constante vers le nord. Le monnayage maurétanien se distingue, à sa naissance, de ses homologues numides par son caractère fortement poliade que l'autorité royale ne concurrence pas ou faiblement, sauf en deux endroits, ŠMŠ (Volubilis ?) et Siga. A la génération suivante, en revanche, les relations des cités et des rois maures semblent se modifier dans un sens qui accrédite l'effacement des unes au profit de la présence royale : seuls dominent désormais au droit des monnaies le nom et l'effigie royale de sorte que l'on ignore la localisation exacte des ateliers : sans doute $\breve{M} \check{S}$ pour Bogud, et Iol pour Bocchus. Ce sont précisément ces mêmes lieux qui continuent d'émettre pendant l'Interrègne (à Iol) et dans le royaume de Juba II et de Ptolémée (Iol-Caesarea et, très subsidiairement, ŠMŠ, sous Juba).

Une question se pose devant l'existence de plusieurs ateliers royaux fonctionnant simultanément en Maurétanie: quelle place exacte occupaient les cités qui les abritaient dans l'économie générale du royaume? S'il s'agissait de regiae, quel sens donner à cette dénomination? Une observation liminaire s'impose : les termes latin (regia) ou grec (baséileion) qui qualifient dans nos sources plusieurs cités numides ne sauraient désigner des capitales au sens strict du terme, c'est-à-dire des cités regroupant, au sein d'un Etat centralisé, toutes les fonctions régaliennes, comme c'était le cas de Rome et celui des capitales du monde hellénistique. La conception encore en partie tribale du pouvoir numide et maure et la fluidité des limites territoriales en excluent l'existence. Ce qui est certain, c'est que les rois numides se sont, à partir de Syphax et de Massinissa, appuyés sur un certain nombre de cités au point que les Italiens et les Grecs ont trouvé en elles des analogies avec les réalités de leur univers politique : les rois y avaient établi une résidence, leur famille, éventuellement un atelier royal, leur trésor, y disposaient de biens, en bref, avaient noué des liens étroits avec plusieurs d'entre elles tout comme ils s'étaient attachés un certain nombre de peuples, appelés pour cela regiani. Cette tendance dénote l'attention nouvelle portée aux cités comme source de richesse fiscale et comme vitrine du pouvoir royal; que deux cités, Siga et Cirta (proches sans doute du berceau des deux tribus principales), se soient finalement détachées du groupe dénote aussi l'attraction du modèle politique dominant en Méditerranée. 
Si l'on se tourne à présent vers la Maurétanie occidentale, on s'aperçoit que jamais aucune cité n'y fut reconnue comme une regia, à quelque période que ce fût: le fait pourrait suggérer que le royaume maure resta longtemps étranger aux mutations qui affectaient les royaumes numides. Cet argument est confirmé par les analyses de J. Alexandropoulos au sujet des premières émissions royales, celles de Bocchus I, montrant que l'autorité royale n'avait alors que faiblement prise sur les cités maures (cf supra). Il n'en reste pas moins que ce dernier se rangea aux pratiques numides dont il avait eu connaissance en annexant une partie du royaume de Jugurtha: il reprit l'atelier de Siga, la regia numide, et implanta un second atelier aux confins de son territoire, en un lieu dit ŠMŠ, éventuellement Volubilis selon J. Alexandropoulos, Gilda pour V. Bridoux (2006).

Cette hypothèse relance la fameuse thèse de Volubilis regia Iubae, soutenue par J. Carcopino puis par A. Jodin à partir d'arguments peu convaincants, mais à laquelle l'argument monétaire vient conférer une nouvelle solidité. Cependant l'absence de traces archéologiques probantes et d'indices autorisant à rattacher Volubilis à un lieudit $\breve{S} M S ̌$ incite à s'en tenir à la prudence et sans doute convient-il de se borner à considérer la position intéressante de la cité, qui était bien de nature à attirer les rois : située dans un bassin très fertile, mais isolée dans une région que dominaient les communautés tribales, Volubilis fournissait aux rois le lieu idéal pour traiter avec les chefs de tribus, comme les gouverneurs de Tingitane le firent bien plus tard à leur tour. A ce titre, elle était bien une regia et le demeura sans doute jusqu'à Juba II et Ptolémée. Mais déjà leur prédécesseur Bocchus II avait élu pour centre unique la cité de Iol, située au centre du royaume et à laquelle Micipsa s'était peut-être lui-même intéressé : la présence (près de Tipasa) du vaste Mausolée de la Chrétienne, qui combine des éléments architecturaux hellénistiques et libyques et qui est le signe d'un pouvoir fort, est attribuée, selon les historiens, à l'un ou l'autre de ces deux souverains. Par la suite, Juba II et Ptolémée n'ont pas eu l'intention de choisir une seconde "capitale ", complémentaire de Iol, que Juba avait rebaptisée Caesarea. Le modèle d'un pouvoir centralisé dominait désormais largement les pratiques et l'idéologie royales : ainsi, les monnaies de Juba émises à ŠMŠ sont-elles très rares et l'évergétisme royal s'est-il exclusivement focalisé sur Caesarea; quant à la splendeur de Volubilis, qui date du II ${ }^{\mathrm{e}}$ siècle ap. J.-C., elle est due à la richesse de ses élites locales.

L'existence d'une capitale unique à partir de laquelle les rois Juba II et Ptolémée régissaient leur immense royaume pose d'emblée la question de la réalité de leur autorité. Plusieurs réponses peuvent y être apportées : elles donnent la mesure des conditions variées auxquelles cette autorité était susceptible de s'appliquer et reflètent le caractère très hétérogène des communautés qui étaient confiées aux souverains. Ces derniers se sont tout d'abord certainement appuyés sur une pratique ancienne du pouvoir royal qui reposait sur la nécessité de déplacements réguliers, avec l'objectif de renouveler en temps voulu la fidélité des tribus : cet usage n'est directement attesté que pour Juba II qui fit plusieurs voyages dans la partie occidentale du royaume, dans l'Atlas et aux îles Canaries (Pline, HN, V, 21-52 et VI, 202), et dont l'objectif scientifique a pu se combiner à des nécessités politiques. En outre, les honneurs dont les deux rois firent l'objet dans les cités ibériques n'ont sans doute pas manqué de susciter des déplacements en Bétique : même si l'embarquement pouvait se faire à partir de Caesarea où était stationnée la flotte royale, un détour par les terres occidentales n'est pas à exclure. Enfin, les guerres menées aux côtés des Romains, à la lisière de l'Africa, 
donnaient l'occasion aux rois de vérifier l'attachement des tribus et des cités dans les régions qu'ils traversaient: les dissidences des Gétules ou des Maures observées à l'occasion de la guerre gétulique (en 6) ou de la révolte de Tacfarinas (17-24) en démontrent l'utilité.

D'autres pratiques, nouvelles celles-ci, permettaient d'assurer la présence royale par le recours à des intermédiaires qui agissaient au nom des rois : on sait, en effet, qu'une administration composée d'esclaves et d'affranchis, sur le modèle de l'administration julio-claudienne, s'était formée à Caesarea : plusieurs tombes et dédicaces funéraires ont été retrouvées dans les nécropoles de la capitale et l'administration provinciale s'est d'abord appuyée sur l'existence de ce personnel ; ce dernier s'était étoffé et peut-être spécialisé à l'initiative de Ptolémée, à propos duquel Tacite souligne la confiance (excessive selon lui) qu'il accordait à ses affranchis (Ann., IV, 23, 1). Leurs tâches devaient les amener à représenter les intérêts du roi en percevant les impôts des cités ou les revenus des biens royaux. On a voulu voir en Aedemon un délégué du roi Ptolémée en Tingitane, ce qui pourrait expliquer sa présence dans la région au moment de la mort du roi, sa révolte contre Rome (Pline, $H N, \mathrm{~V}, 11,1$ évoque sa fidélité à la mémoire de son maître) et sa capacité à diriger l'armée royale comme à s'assurer l'alliance du chef Salabos. Le dernier royaume de Maurétanie manifeste l'apparition de structures administratives de pouvoir dont le modèle est à chercher à Rome et non dans le passé africain : même si Masinissa et Micipsa semblent avoir implanté un contrôle assez serré en Numidie orientale, en y créant des circonscriptions et des cités regiae (Bridoux : 247-256), leur tentative resta limitée à cette région que Masinissa avait eu intérêt à tenir solidement face à Carthage ; elle ne semble pas avoir donné naissance à un système administratif organisé à l'échelle du royaume et l'on observe que les principaux acteurs du pouvoir numide restèrent les parents du roi.

Abordons, pour finir, le cas de cet autre levier du pouvoir royal qu'était l'armée. Les armées africaines furent longtemps marquées du sceau des réalités tribales: les rois fédéraient autour d'eux, pour des actions ponctuelles, un certain nombre de chefs qui amenaient avec eux leurs guerriers. Syphax recrute des unités de combat dans la région de Tétouan, Jugurtha renouvelle son armée auprès des Gétules, tandis que d'autres Gétules, ligués autour de Juba I font défection pour rallier le camp de César. Ces coalitions éphémères sont attestées jusqu'à l'époque du roi Juba II à qui échappe le contrôle des tribus situées aux sud de l'Ampsaga. Les tribus gétules de son royaume se liguent à celles de la province lors du conflit où s'illustra L. Cornélius Balbus (21/20) ou lors de la "guerre gétulique »; à nouveau plusieurs d'entre elles, autour d'un certain Mazippa, s'engagent aux côtés de Tacfarinas qui put ainsi regrouper des Musulames, des Cinithiens et des Maures (Tacite, Annales, II, 52); d'autres Maures, en revanche, suivent le roi Ptolémée qui vient soutenir les Romains en 24 et les Romains les laissent pratiquer la razzia, sous le commandement de leurs chefs ; on peut également supposer que les voyages de Juba II en Maurétanie occidentale avaient, outre leur intérêt scientifique, celui de renouveler les liens d'allégeance des tribus maures. La puissance guerrière des souverains devaient donc beaucoup à cette capacité de renouvellement permanent de leurs armées qui, de plus, combattaient les Romains avec des pratiques souvent déroutantes pour ces derniers. Mais ces alliances faisaient aussi leur faiblesse puisque les tribus n'étaient tenues que par l'intérêt du butin et pouvaient se rallier à une autorité qui leur semblait plus forte que celle du roi: elles étaient, structurellement ou occasionnellement, dissidentes. 
Les sources attestent cependant, à partir de la $\mathrm{II}^{\mathrm{e}}$ Guerre punique, l'évolution de ces armées royales: leur composition, leur équipement, leurs techniques de guerre se modifient en même temps que s'impose progressivement le concept d'une armée (ou d'un corps) permanente. Syphax avait engagé un centurion romain pour organiser et former son armée "à la romaine ", mais il connaissait déjà les principes de la guerre grecque par l'entremise des Carthaginois, tout comme Masinissa qui s'était très probablement adjoint les services de spécialistes grecs et italiens; tous deux possédaient aussi une flotte. Jugurtha est formé dans l'état-major de Scipion de sorte que, pendant la guerre contre Rome, il est en mesure d'entraîner ses troupes à la romaine. Les informations concernent pour l'essentiel les armées numides, mais l'on sait que la circulation des connaissances était rapide et que, si le Maure Bocchus I put opposer un interprète à celui de Scipion (Sall., Jug., CIX, 4), c'est qu'il était informé des modalités de la diplomatie romaine et savait les mettre en pratique. Ses lointains successeurs sur le trône de Maurétanie, Juba II et Ptolémée, possédaient eux aussi une armée composite et en partie du moins permanente, inspirée par l'armée romaine : si Juba II avait créé des cohortes urbaines à Caesarea, il avait très certainement repris aussi l'armée de son père dont Fr. Bertrandy (1988) et Y. Le Bohec (2004) ont mis en évidence les caractères : il s'agissait d'une armée «moderne », présentant un visage analogue à toutes les armées de son temps; formée à la fois d'unités tribales qui maintenaient les coutumes guerrières africaines, encadraient les éléphants et fournissaient une forte cavalerie; d'une garde royale de mercenaires; de légions rompues à toutes les tactiques de l'art guerrier (renseignement, combat au corps à corps, poliorcétique, stratagèmes) au point d'inquiéter les armées de César.

C'est à cette armée composite (en tout cas à une fraction) dont l'affranchi royal Aedemon prit la tête, que se heurtèrent les armées romaines envoyées en Maurétanie pour l'annexer: Aedemon s'appuyait à la fois sur des unités sachant combattre les Romains sur leur propre terrain et sur des forces auxiliaires, celles de tribus, en particulier les troupes menées par le chef Salabos (issu de l'Atlas). Il ne semble pas, en revanche, avoir séduit les cités, comme l'indiquent la résistance que Volubilis lui opposa ainsi que les nombreuses promotions de cités aux lendemains de l'annexion, aussi bien en Césarienne qu'en Tingitane. On connaît certains détails des opérations et le panorama général des forces en présence uniquement dans la future Tingitane, ce qui laisse à penser que la crise s'est surtout déroulée dans cette partie du royaume ; comparativement, et malgré la présence de trois trésors monétaires (indices d'une situation troublée), la future Césarienne semble avoir été relativement épargnée. Les aptitudes réelles de l'armée royale, qui avait déjà fait ses preuves dans plusieurs guerres ayant touché les confins du royaume et de la province (Coltelloni-Trannoy, EB, XV, J13 : 3928), légitimaient bien l'importance des forces engagées par Rome contre Aedemon: la légion X Gemina et de nombreuses unités auxiliaires débarquèrent d'Espagne, le proconsul de Bétique Umbonius Silo étant chargé de l'approvisionnement des troupes (DC, LX, 24); s'y ajoutaient les corps levés par les colonies romaines et les cités maures de Tingitane (Rebuffat 1997). Le bellum s'acheva avant l'avènement de Claude, c'est-à-dire avant le 21 janvier 41 , de sorte que la conquête du royaume ne dura pas plus d'une année. Elle fut suivie d'une autre guerre, contre les tribus maures de Salabos, en 41-42, poursuivies dans l'Atlas par Suetonius Paulinus, puis jusque dans les déserts par Hosidius Geta, qui les amena à accepter ses conditions (DC, LX, 9, 1-5). 


\section{L'ouverture au monde méditerranéen}

31 Les mutations qui ont infléchi les royaumes maures et numides vers des monarchies de type hellénistique se sont accompagnées d'un processus d'ouverture au monde méditerranéen qui en fut même la condition première: celui-ci a pris la forme de relations diplomatiques et militaires avec les puissances dominantes du temps, Carthage, Rome, les royaumes et les cités helléniques, accompagnées d'une participation active aux échanges commerciaux.

32 Les royaumes maures ont ceci d'original que les deux manifestations principales de ce processus (politique et économique) ont obéi à des rythmes différents selon les époques. A l'époque la plus ancienne, on observe un décalage assez marqué entre leur insertion précoce dans les courants économiques méditerranéens et leur intégration aux alliances et aux conflits qui affectèrent l'espace méditerranéen. En effet, bien que ces royaumes aient abrité des cités de Libyphéniciens au même titre que les royaumes numides, et que les produits ibériques et puniques se retrouvent en abondance sur les sites côtiers de Maurétanie occidentale, ils ont toutefois conservé une certaine distance vis-à-vis de Carthage : on ne note, de la part des rois maures, ni alliances matrimoniales avec les aristocrates puniques, ni contributions militaires pendant les guerres puniques, si ce n'est le soutien de Baga à la cause de Massinissa. Ils n'ont pas davantage établi de contacts diplomatiques ou financiers avec les puissances grecques comme l'ont pratiqué les rois numides, sauf à l'extrême fin de la période, du fait de Juba II et de Ptolémée.

33 En revanche, la période ultérieure se caractérise par une convergence assez nette entre le processus économique et le processus politique. Les successeurs de Baga restèrent étrangers jusqu'à Bocchus I à l'emprise romaine qui s'était mise en place dans les royaumes numides à partir de Masinissa. Bocchus fut en effet le premier souverain africain à lier ses ambitions personnelles aux intérêts de Rome à l'occasion de la fameuse tractation qui eut lieu entre lui et Sylla: le roi maure accepta de livrer Jugurtha contre une partie de son royaume. On voit par cet exemple quels bénéfices considérables les rois pouvaient retirer de l'alliance romaine. L'événement eut pour conséquence immédiate de lier Bocchus et les siens à Sylla et à la faction des optimates. Les liens restèrent solides en dépit d'une tentative probable des marianistes pour contacter les rois maures, dont témoigne l'équipée de Sertorius en Maurétanie; la présence des populaires fut néanmoins fugace en raison de l'intervention de Pompée qui rendit au roi Ascalis sa principauté de Tingi (Plutarque, Sertorius, 9 et Pompée, 12 ; Callegarin, 2002: 12-20). Cette tradition d'allégeance aux optimates, et notamment à Pompée, persista jusqu'à ce que les rois maures Bogud et Bocchus II aient décidé de soutenir César, le chef des populares, au lieu de Pompée, auquel s'était allié leur ennemi Juba I. Par la suite, leurs options prirent des voies divergentes: Bocchus, qui avait gagné un avantage tangible à soutenir César, resta fidèle à son fils, Octave ; Bogud, sans doute inquiet de la puissance nouvelle de son voisin, prit le parti d'Antoine, au moment même où ce dernier gagnait le soutien du gouverneur de Bétique, Asinius Pollion, et de son questeur, L. Cornelius Balbus, membre d'une grande famille de Gadès, réfugié auprès de Bogud après s'être rendu coupable d'exactions (Cic., Af Fam., X, 33). Ces trois soutiens quasi simultanés suggèrent l'existence de négociations entre eux et Antoine, où l'on devine le poids du proconsul et les liens privilégiés qui s'étaient tissés entre la dynastie royale et les familles gaditaines. Les relations entre les Maures et l'Espagne, 
qui étaient anciennes (parfois conflictuelles), se maintiennent plus tard sous une forme nouvelle quand Juba II puis son fils deviennent magistrats honorifiques de Gadès et de Carthagène.

Ces relations personnelles de clientèle rehaussaient d'une part le prestige des familles romaines et contribuaient à en conforter la puissance ; elles médiatisaient d'autre part les relations politiques des rois avec le sénat romain qui concluait régulièrement des alliances avec eux et les qualifiait du titre officiel de rex amicus et socius. Ces alliances, avec les titres et les insignes qui les accompagnaient, procuraient aux rois l'avantage de soutiens puissants, dont ils savaient bien profiter à l'occasion, mais elles les plaçaient aussi dans une position de subordination implicite : elles les contraignaient à prendre parti lors des guerres civiles, nous l'avons vu plus haut, ou bien à accepter des Romains des exigences variées (levées de troupes, livraisons de céréales ou de fauves, cession de terres pour les fondations coloniales dans le royaume de Juba II). Tout en tirant parti du soutien militaire et logistique des rois maures et numides, Rome assurait la stabilité des royaumes et confortait une lignée au détriment d'une autre.

La proximité entre les rois de Maurétanie et leurs protecteurs romains s'accentua encore à l'époque impériale. Juba II était un véritable prince romain par son éducation, par son mariage qui l'apparentait à la famille impériale, par sa citoyenneté romaine enfin qu'il avait reçue d'Auguste sans doute et que son épouse détenait aussi: de la sorte, leur fils Ptolémée était un citoyen de naissance. Proximité des familles, proximité accrue du royaume avec l'Empire aussi. Non seulement l'empereur avait prélevé des terres en Maurétanie pour fonder treize colonies romaines (qui venaient compléter les fondations d'Afrique proconsulaire), mais elles étaient rattachées administrativement à la Bétique et sans doute à la Tarraconaise et à l'Africa, de même que le conuentus de citoyens romains établi à Icosium dépendait peut-être d'Ilici (Tarraconaise). On sait aussi par le cas des Thudedenses (AE, 1985, 972) que la pratique de la limitatio avait cours dans le royaume et que cette limitatio couplée à une immunité fiscale avait été garantie à la communauté maurétanienne au nom de l'empereur et sous le contrôle du roi : ce dernier agissait ni plus ni moins qu'un gouverneur. Cette situation préfigurait l'annexion du royaume et l'octroi de statuts romains à des cités maurétaniennes dès les lendemains de la conquête. Du point de vue symbolique aussi, le mariage de Juba avec Cléopâtre Séléné établissait des connexions évidentes entre le royaume maurétanien et l'ancien royaume lagide, désormais province romaine, entre des familles naguère encore ennemies, désormais réunies sous l'autorité impériale. Du point de vue institutionnel, enfin, on observe la sacralisation des souverains qui répond à celle de l'empereur: Juba II crée le culte impérial à Caesarea tandis que le culte rendu à Ptolémée emprunte aux formes romaines du culte impérial. Au total, l'imitatio imperii est poussée assez loin, par laquelle les rois confortaient leur autorité locale en reproduisant certaines manifestations du pouvoir impérial.

Le partenariat politique qui s'instaura peu à peu entre les royaumes maures et l'Empire romain eut son équivalent économique, mais il est mal connu en raison des recherches qui sont lacunaires et inégales selon les sites. Je reprends ici les conclusions qu'a proposées V. Bridoux, à qui l'on doit une synthèse récente en la matière (non encore publiée). Le processus d'insertion de la Maurétanie dans les circuits économiques de l'Empire s'est fait apparemment selon trois temps et fut plus tardif que celui que l'on observe dans les royaumes numides. La première époque, qui va de Baga à Bocchus I, se signale par la rareté des produits italiens en Maurétanie occidentale et leur localisation 
exclusive aux sites méditerranéens et à Lixus. La guerre de Jugurtha, qui eut pour conséquence des mutations profondes dans le royaume de Maurétanie, constitue une époque charnière: tandis que les ports de l'Atlantique et les cités de l'intérieur accueillent les premières importations italiques à la fin $\mathrm{II}^{\mathrm{e}} /$ début $\mathrm{I}^{\mathrm{er}}$ siècle, la variété et l'abondance de ces produits s'accentuent à partir du milieu $\mathrm{I}^{\mathrm{er}}$ siècle av. J.-C. Si la Maurétanie continue d'être un membre actif du "circuit du Détroit», elle l'est désormais autrement: les négociants italiens s'implantent dans le royaume et concurrencent les commerçants ibéro-puniques.

Au milieu du premier siècle av. J.-C., s'amorce une nouvelle phase économique qui va de pair avec l'implication des rois Bogud et Bocchus II dans les guerres romaines et la transformation de Tingi en municipe romain: le marché est désormais dominé par le répertoire italien qui se signale par la standardisation des produits. A l'époque de Juba et de Ptolémée, le site de Mogador reprend la fabrication de la pourpre qui avait été intense à l'époque phénico-punique, de même que les usines de salaison ouvrent dans le nord du Maroc : cela ne signifie pas pour autant que les dérivés du poisson n'aient pas été exploités auparavant, mais qu'ils l'étaient autrement et, sans doute, dans des structures non permanentes. Des projets urbanistiques novateurs, sur le modèle grécoromain connu par les colonies voisines, par les cités ibériques et par les réalisations en cours à Iol-Caesarea, voient le jour à Zilil, Lixus, Sala, Thamusida, Volubilis. Une riche clientèle, qui tire ses revenus de sa participation aux nouveaux réseaux commerciaux, y consomme le vin italien et utilise la vaisselle italique. Ajoutons qu'elle s'initie aussi au latin, ce que suggèrent l'apparition des premiers citoyens d'origine indigène dans les cités maures puis le recours aux pratiques épigraphiques dès les premières années de l'annexion : autant de faits qui apparentent les élites de Maurétanie occidentale à celle des cités orientales, sensibles aux influences gréco-romaines depuis déjà Massinissa. Pour autant, les langues du royaume demeurent le libyque ou le punique, sauf de rares dédicaces rédigées en latin à Caesarea ou dans sa région, et les dieux romains ne sont présents que dans la regia. Ces limites manifestes à la « romanisation » culturelle, même observées dans les cités les plus dynamiques du royaume, donnent la mesure du décalage qui existait entre la participation du royaume à l'Empire dans les sphères politique et économique d'une part, et l'adoption des normes de la vie romaine d'autre part.

Lorsque les Romains installèrent leur administration provinciale à Caesarea, en 42, ils trouvèrent une situation contrastée: un territoire ayant déjà expérimenté certaines formes de l'administration romaine; des cités prêtes à s'engager plus avant dans la voie des échanges avec l'Empire ; des campagnes connaissant déjà les normes de production et de commercialisation exigées par les marchés méditerranéens; des élites municipales intéressées par les pratiques sociales et politiques du monde romain. Mais des communautés nombreuses, des pans entiers du territoire, demeuraient en marge de ces mutations : la Maurétanie était bien encore une terre de confins. 


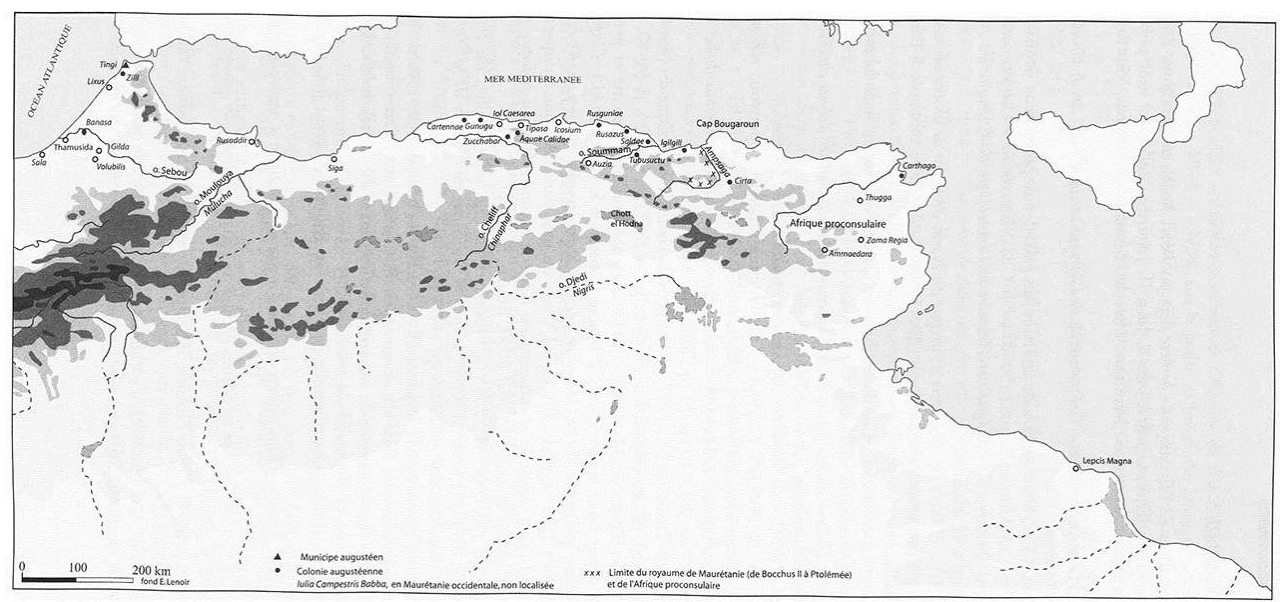

CARTe de LA maurétanie de Juba II et Ptolémée.

\section{BIBLIOGRAPHIE}

Voir les notices de l'Encyclopédie berbère déjà publiées : Aedemon, Bocchus, Bogud, Caesarea, Cirta, Cités, Gétules, Juba I, Juba II, Jugurtha ; celles qui sont publiées dans ce volume : Masaesyles, Masinissa, Massyles, Mastanabal, Micipsa, Musulames.

ALEXANDROPOULOS, 2000 - Les monnaies de l'Afrique antique (400 av. J.-C.-40 ap. J.-C.), Toulouse.

BERTRANDY Fr., 1988 - « L'aide militaire de Juba I aux pompéiens pendant la guerre civile en Afrique du nord (50-46 av. J.-C.) », Actes du $113^{e}$ Congrès national des Sociétés savantes, IV colloque sur l'histoire et l'archéologie de l'Afrique du Nord, Strasbourg, t. II, p. 289-297.

BRIDOUX V., 2006 - Les royaumes d'Afrique du Nord, de la fin de la seconde Guerre punique à la mort du roi Bocchus II (201-33 av. J.-C.), thèse soutenue à Paris I, (non publiée).

CALLEGARIN L., 2002 - « Considérations sur le périple sertorien dans la zone du détroit de Gibraltar (81-78 av. J.-C.) », Pallas, 60, p. 11-43.

CAMPS G., 1961 - Aux origines de la Berbérie. Massinissa ou les débuts de l'histoire (Libyca ArchéologieEpigraphie, VIII, 1) Alger.

CAMPS G., 2007 - Les Berbères. Mémoire et identité, Arles, (4e rééd.).

COARELLI F. \& THÉBERT Y., 1988 - « Architecture funéraire et pouvoir : réflexions sur l'hellénisme numide », MEFR, 100, 2, p. 761-818.

COLTELLoni-tRANNoy M., 1997 - Le royaume de Maurétanie sous Juba II et Ptolémée (25 av. J.-C.-40 ap. J.C.), Paris.

Coltelloni-TRANNoy M., 1997 - « Les liens de clientèle en Afrique du Nord, du II ${ }^{\mathrm{e}}$ siècle av. J.-C. jusqu'au début du principat », BCTHS, n.s., 24, p. 59-82. 
COLTELLONI-TRANNOY M., 2002 - « Hercule en Maurétanie : mythe et géographie au début du principat », in $\mathrm{Cl}$. Briand-Ponsart et S. Crogiez (éd.), L'Afrique du Nord antique et médiévale. Mémoire, identité et imaginaire, Rouen, p. 41-58.

COLTELloni-TRANNoy M., 2005 - «L'usage du grec dans l'Afrique antique », in Cl. Briand (dir.), Identités et cultures dans l'Algérie antique, Actes du colloque organisé par l'Université de Rouen, mai 2003, Rouen, p. 69-117.

COLTELLONI-TRANNOY M., 2005 - « Rome et les rois amis et alliés du Peuple romain en Afrique ( $\mathrm{I}^{\mathrm{er}} \mathrm{s}$. av. J.-C.--Ir ${ }^{\text {er }}$ s. ap. J.-C.) », Actes du colloque de la SOPHAU, Poitiers, 1-3 avril 2005, Pallas, 68, p. 117-144. COLTELLONI-TRANNOY M., (à paraître) - « Guerre et circulation des savoirs : le cas des armées numides ", in J.-Ch. Couvenhes, S. Crouzet et S. Péré-Noguès (éd.), Pratiques et identités culturelles des armées hellénistiques du monde méditerranéen, Actes du colloque de Tours (23-24 mars 2007).

DESANGES J., 1957 - « Le triomphe de Cornelius Balbus (19 avant J.-C.) », Revue Africaine, CI, p. 5-43. DESANGES J., 1960 - « Mauretania ulterior Tingitana », Bulletin d'Archéologie marocaine, IV, p. 437-441. DESANGES J., 1964 - « Les territoires gétules de Juba II », Revue des Études anciennes, LXVI, p. 33-47. DESANGES J., 1980 - Pline l'Ancien, Histoire naturelle, V, 1-46, Edition, traduction et notes, Paris. GHAZI-BEN MAÏsSA H., 1993 - «Volubilis et le problème de regia Iubae », l'Africa romana, X, 1, p. 243-261.

GSELL St., 1913-1928 - Histoire ancienne de l'Afrique du Nord, Paris (= HAAN).

HAMDOUNE Ch., 1994 - « Note sur le statut colonial de Lixus et de Tanger », Antiquités africaines, 30, p. 81-87.

HAMDOUNE Ch., 2003 - «Y a-t-il eu des déplacements contraints de population en Afrique romaine? », Travaux et recherches de l'umLV, Littératures, Sciences humaines, 7, p. 13-37.

LASSERE J.-M., 1979 - « Onomastica Africana I-IV », Antiquités africaines, 13, p. 227-234.

LE BOHEC Y., 2004 - «L'expédition de Curion en Afrique : étude d'histoire militaire », in l'Africa romana, XV, p. 1603-1616.

LE BOHEC Y., (à paraître) - « L'armée de Numidie au temps de Juba $\mathrm{I}^{\mathrm{er}}$. »

LEVEAU Ph., 1984 - Caesarea de Maurétanie, une ville romaine et ses campagnes, Rome (CEFR 70).

LEVÊQUE P., 1999 - « Avant et après les princes. L'Afrique mineure de l'Age du fer », Les princes de la protohistoire et l'émergence de l'Etat, Actes de la table ronde internationale de Naples (1994), Naples (CEFR 252), p. 153-164.

REBUFFAT R., 1997 - « Romana arma primum Claudio principe in Mauretania bellauere », in Y. Burnand, Y. Le Bohec, J.-P. Martin (éds), Claude de Lyon, empereur romain. Actes du colloque ParisNancy-Lyon, nov. 1992, Paris, p. 277-320.

ROLLER, D. W., 2003 - The World of Juba II and Kleopatra Selene, New York and London.

SMADJA, E., 1983 - « Modes de contact, sociétés indigènes et formation de l'Etat numide au II ${ }^{\mathrm{e}} \mathrm{s}$. av. n. ère ", Modes de contact et processus de transformation dans les sociétés anciennes (CEFR 67), Rome, p. 685-702. 
INDEX

Mots-clés : Anthropologie, Antiquité, Géographie, Histoire, Histoire politique, Maurétanie 